\title{
Modified composite material developed on the basis of no-fines asphalt concrete
}

\author{
Andrey Mikhasek ${ }^{1, *}$, and Boris Ivanov ${ }^{1}$ \\ ${ }^{1}$ Samara State Technical University, Institute of Architecture and Civil Engineering, \\ Molodogvardeyskaya St., 194, Samara, 443001, Russia
}

\begin{abstract}
Being a composite material, asphalt concrete is widely used in hydraulic engineering and road construction. The paper proves one of asphalt concrete modification, which includes first creating a skeleton of no-fines concrete and then its washing-down with bituminous materials by a hot procedure, can be successfully used in hydraulic structures Modified composite material based on no-fines asphalt concrete has a harder skeleton because of links from cement stone and has a technological advantage, as through the proposed technology it allows to reduce the cost of filling porous spaces. This technology allows to conclude that concrete aggregate with size fractions of $120 \mathrm{~mm}$ or less and frost resistance of 50 cycles and less can be recommended for fastening of slopes.
\end{abstract}

\section{Introduction}

The use of composite materials in construction allows to build constructions with preprojected specified properties. Concrete and asphalt concrete as its type [1-5] can be named among the most common composite materials. In asphalt concrete bituminous materials perform the fiction of a binder. In hydraulic engineering and road construction asphalt concrete became widely spread and is often used as a protective coating.

Asphalt concrete quality is affected by the selection of its optimal composition and primarily depends on the quality of the larger aggregate, and the availability of silt particles, their size, shape and roundness [1]. Thus, a large number of requirements to raw materials, leads to an increase in the number of operations, in financial costs and in construction time.

In addition, sometimes there might not be enough necessary materials on a certain construction site. That leads in its turn to the use of transported expensive materials.

In hydraulic engineering, the size (or fineness) of construction stone to be used in coast protecting structures at major watercourses such as the Volga, ranges from 300 to $600 \mathrm{~mm}$ [6]. Shipping companies often refuse to transfer such materials because road transport with carrying capacity of more than 25 tons cannot be used on public roads, and the distance from the quarry to the construction site can be rather long and does not allow to use equipment outside the quarry. Often loading in dump trucks at the quarry is carried out with an increasing share of overburden to reduce shock loads in the process of unloading the

*Corresponding author: andremixas@mail.ru 
stone material out of a bucket excavator. Sometimes it requires special loading nodes for reloading by use of lower-capacity hydraulic excavators. These extra actions increases selling price of stone materials. Railway and water transport also require special actions when performing loading operations. Thus a possible "transport" dimension of materials does not exceed $250 \mathrm{~mm}$ and the optimal value involves the use of stone materials of 80 $120 \mathrm{~mm}$ fineness.

These restrictions resulted in the use of more expensive construction solutions compared to rubblework, i.e. mesh structures, monolithic steel-concrete and concrete slabs, concrete oversize (tetrapods, tetrahedrons, stars, cubes, etc.), concrete-filled mates, polymer concrete, asphalt concrete [7-10].

\section{Materials and Methods}

Asphalt concrete is a traditional material for bank protection. However, it has a number of shortcomings related to its reliability in the case of ageing of bituminous binder and using large filler fractions of $120 \mathrm{~mm}$ or less.

To protect asphalt concrete from destruction, we propose a new technology that provides first making a skeleton of no-fines concrete and then washing-down with bituminous materials by a hot procedure. This material is shown on Figure 1.
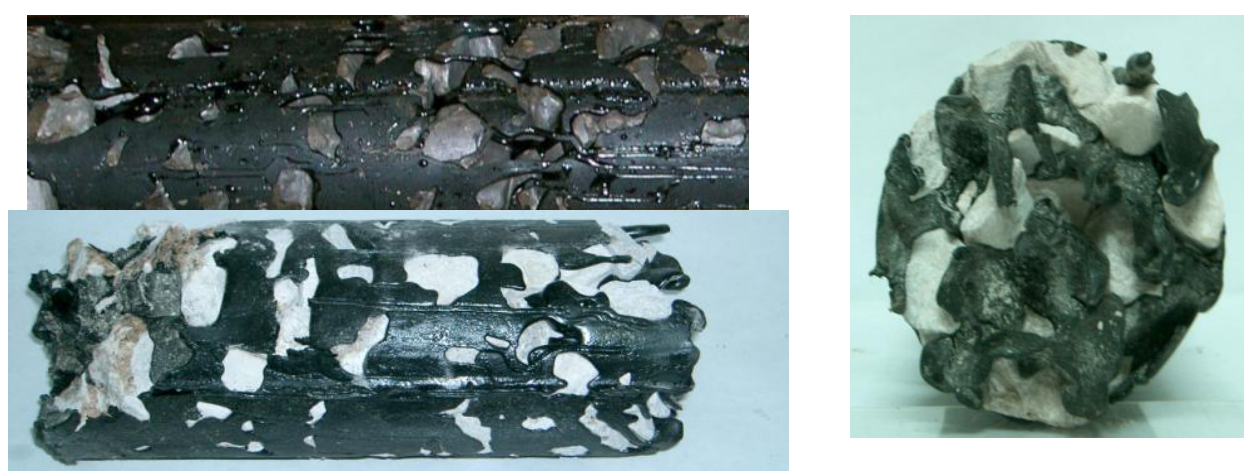

Fig. 1. Sample view

Besides, this technology has the advantage when compared to traditional concrete plates because higher demands to the preparation of the foundation (variations in the horizon should not exceed 2-3 cm for every 5 meters of a slope) are put forward when laying prefabricated monolithic plates. The use of this technology makes it possible to abandon the increased requirements to the preparation of a foundation. Hollows under the plates can be filled with bitumen in the process of filling the porous space, its excess expenditure not more than $6 \%$.

To make no-fines concrete we recommend using the following materials:

- gravel mark 400-600;

- cement - M400-D20 with discharge of $150 \mathrm{~kg} / \mathrm{m}^{3}$;

- water $\mathrm{W} / \mathrm{TS}=0.45$.

When using gravel of 20-40 fineness, the authors defined strength of no-fines concrete during laboratory tests. Its average strength is $45-50 \mathrm{~kg} / \mathrm{m}^{3}$. The researchers also studied changes in frost resistance and demonstrated that there was an increase in the number of cycles $10-15$ on average. 


\section{Results}

For the implementation of the proposed technology it is required to standardize the temperature of bituminous mixture according to the composition of bitumen, aggregate size and thickness of the bind. Temperature selection is conducted by function (1) received as a result of laboratory and field studies:

$$
h=d \cdot\left(\ln \frac{G a}{B \cdot \Delta K}\right) \cdot A \cdot m,
$$

where $\mathrm{d}$ is an effective diameter of the particle of porous medium,

GA - Galileo criterion - is defined by function (2)

$$
G a=\frac{\operatorname{Re}^{2}}{F r}=\frac{g \cdot d^{3}}{v^{2}}
$$

$\Delta K=\frac{K_{n}}{K_{z}}-$ the ratio of the temperature at the beginning of $K n$ supply to the temperature of the fluid hanging $\mathrm{Kz}$ (for water is equal to $273 \mathrm{k}$, for bitumen - $373 \mathrm{~K}$ ); m - coefficient of porosity, defined empirically $0.35-0.45$, A and B are correction coefficient found by the least squares method based on the results of the pilot data processing.

The values of the coefficients A and B depend on the permanent indicators of porous medium $\mathrm{d}$ and $\mathrm{m}$ and are defined by the equations (3) and (4).

$$
\begin{aligned}
& A=-0.39 \cdot \frac{m}{d}+13.2 \\
& B=2460 \cdot \frac{d}{m}-60 .
\end{aligned}
$$

Taking into consideration that we recommended to use stone with $100 \mathrm{~mm}$ fineness. According to the calculation methodology we made technological calculations of the temperature of laying, which are presented in Tables 1 and 2.

Table 1. The relationship between Galileo criterion, aggregate fineness and bitumen mark

\begin{tabular}{|c|c|c|c|c|c|c|c|}
\hline Bitumen & Aggregate & \multicolumn{7}{|c|}{ Technological delivery temperature, ${ }^{\circ} \mathbf{C}$} \\
\cline { 2 - 8 } mark & fineness & $\mathbf{1 2 0}$ & $\mathbf{1 4 0}$ & $\mathbf{1 6 0}$ & $\mathbf{1 8 0}$ & $\mathbf{2 0 0}$ & $\mathbf{2 2 0}$ \\
\hline \multirow{4}{*}{ BN-IIU } & $0.02-0.08$ & 8390 & 30175 & 108530 & 390343 & 1403922 & 5049402 \\
\cline { 2 - 8 } & $0.02-0.1$ & 14498 & 52143 & 187540 & 674512 & 2425977 & 8725366 \\
\cline { 2 - 8 } & $0.04-0.1$ & 23022 & 82801 & 297806 & 1071100 & 3852362 & 13855558 \\
\cline { 2 - 8 } & $0.08-1.0$ & 48930 & 175983 & 632946 & 2276479 & 8187673 & 29448110 \\
\hline \multirow{4}{*}{ BND } & $0.02-0.08$ & 3372 & 13137 & 51185 & 199428 & 777010 & 3027380 \\
\cline { 2 - 8 } 60/90 & $0.02-0.1$ & 5827 & 22701 & 88448 & 344611 & 1342673 & 5231313 \\
\cline { 2 - 8 } & $0.04-0.1$ & 9252 & 36049 & 140453 & 547230 & 2132115 & 8307131 \\
\cline { 2 - 8 } & $0.08-1.0$ & 19664 & 76617 & 298513 & 1163064 & 4531521 & 17655681 \\
\hline \multirow{4}{*}{ BN-III } & $0.02-0.08$ & 3285 & 13322 & 54022 & 219070 & 888374 & 3602534 \\
\cline { 2 - 8 } & $0.02-0.1$ & 5677 & 23020 & 93350 & 378553 & 1535110 & 6225178 \\
\cline { 2 - 8 } & $0.04-0.1$ & 9014 & 36555 & 148237 & 601129 & 2437698 & 9885352 \\
\hline \multirow{4}{*}{ BND } & $0.08-1.0$ & 19159 & 77692 & 315057 & 1277618 & 5180996 & 21009976 \\
\cline { 2 - 8 } & $0.02-0.08$ & 1626 & 7144 & 31383 & 137863 & 605626 & 2660483 \\
\cline { 2 - 8 } & $0.02-0.1$ & 2810 & 12345 & 54230 & 238228 & 1046522 & 4597315 \\
\cline { 2 - 8 } & $0.04-0.1$ & 4462 & 19603 & 86115 & 378297 & 1661839 & 7300366 \\
\cline { 2 - 8 } & $0.08-1.0$ & 9484 & 41663 & 183025 & 804019 & 3532013 & 15515939 \\
\hline \multirow{4}{*}{ BN-IIIU } & $0.02-0.08$ & 2112 & 8227 & 32055 & 124891 & 486598 & 1895880 \\
\cline { 2 - 8 } & $0.02-0.1$ & 3649 & 14216 & 55390 & 215811 & 840841 & 3276080 \\
\cline { 2 - 8 } & $0.04-0.1$ & 5794 & 22575 & 87958 & 342700 & 1335225 & 5202294 \\
\cline { 2 - 7 } & $0.08-1.0$ & 12315 & 47981 & 186942 & 728362 & 2837840 & 11056771 \\
\hline
\end{tabular}


Table 2. The relationship between Galileo criterion, aggregate fineness, bitumen mark and penetration depth

\begin{tabular}{|c|c|c|c|c|c|c|}
\hline \multirow{2}{*}{$\ln (\mathbf{G a} / \mathbf{d k} \times \mathbf{B}) \times \mathbf{A}$} & \multicolumn{7}{|c|}{ Multiplication $\mathbf{d} \times \mathbf{m}$} \\
\cline { 2 - 7 } & $\mathbf{0 . 0 1}$ & $\mathbf{0 . 0 2}$ & $\mathbf{0 . 0 3}$ & $\mathbf{0 . 0 4}$ & $\mathbf{0 . 0 5}$ & $\mathbf{0 . 0 6}$ \\
\hline 5 & 0.05 & 0.1 & 0.15 & 0.2 & 0.25 & 0.3 \\
\hline 10 & 0.1 & 0.2 & 0.3 & 0.4 & 0.5 & 0.6 \\
\hline 15 & 0.15 & 0.3 & 0.45 & 0.6 & 0.75 & 0.9 \\
\hline 20 & 0.2 & 0.4 & 0.6 & 0.8 & 1 & 1.2 \\
\hline 25 & 0.25 & 0.5 & 0.75 & 1 & 1.25 & 1.5 \\
\hline 30 & 0.3 & 0.6 & 0.9 & 1.2 & 1.5 & 1.8 \\
\hline 35 & 0.35 & 0.7 & 1.05 & 1.4 & 1.75 & 2.1 \\
\hline 40 & 0.4 & 0.8 & 1.2 & 1.6 & 2 & 2.4 \\
\hline 45 & 0.45 & 0.9 & 1.35 & 1.8 & 2.25 & 2.7 \\
\hline 50 & 0.5 & 1 & 1.5 & 2 & 2.5 & 3 \\
\hline 55 & 0.55 & 1.1 & 1.65 & 2.2 & 2.75 & 3.3 \\
\hline 60 & 0.6 & 1.2 & 1.8 & 2.4 & 3 & 3.6 \\
\hline 65 & 0.65 & 1.3 & 1.95 & 2.6 & 3.25 & 3.9 \\
\hline 70 & 0.7 & 1.4 & 2.1 & 2.8 & 3.5 & 4.2 \\
\hline 75 & 0.75 & 1.5 & 2.25 & 3 & 3.75 & 4.5 \\
\hline 80 & 0.8 & 1.6 & 2.4 & 3.2 & 4 & 4.8 \\
\hline 85 & 0.85 & 1.7 & 2.55 & 3.4 & 4.25 & 5.1 \\
\hline 90 & 0.9 & 1.8 & 2.7 & 3.6 & 4.5 & 5.4 \\
\hline 95 & 0.95 & 1.9 & 2.85 & 3.8 & 4.75 & 5.7 \\
\hline 100 & 1 & 2 & 3 & 4 & 5 & 6 \\
\hline 105 & 1.05 & 2.1 & 3.15 & 4.2 & 5.25 & 6.3 \\
\hline 110 & 1.1 & 2.2 & 3.3 & 4.4 & 5.5 & 6.6 \\
\hline
\end{tabular}

Basing our analysis on Tables 1 and 2, for the implementation of the proposed technology we recommend that technological temperature of use should be $170-180{ }^{\circ} \mathrm{C}$ for no-fines concrete with $80-120 \mathrm{~mm}$ aggregate (with a safe temperature of $220^{\circ} \mathrm{C}$ ).

\section{Discussion}

The experiments demonstrated that it is not enough to use only no-fines concrete to improve freeze resistance of the composite material. Laboratory tests of polymer concrete showed that their use leads to a significant increase of frost resistance, but also leads to a 4times increase in the cost of polymer concrete compared to no-fines concrete cost. In addition, there are higher demands to the process of polymer concrete laying connected with the time it requires to set with a binder thus making it obligatory to prepare and unload it together with large aggregates at the construction site. All this leads to an increase in the cost of works and requires the purchase of special equipment. In this regard, we propose to replace polymeric binder by the bitumen. We chose a hot filling technology directly on the site as a means to deliver bituminous materials. This technology has a number of technological and structural advantages. Firstly, washing-down with bituminous materials makes it possible to get the final product in a short time, because the asphalt quickly cools and becomes solid. To warm bituminous material up and to deliver it to the point of laying, standard equipment (like bitumen heaters used in road construction) is used. Secondly, additional processing of no-fines concrete with bitumen material will significantly increase frost resistance of the final product. It will allow the use of stone materials with frost resistance of 50 cycles and less. Thirdly, standardizing temperature and place of bituminous material delivery, it is possible to make a layer of drainage and drainage water release that will reduce the thickness of the bind and also reduce costs. 
We assume that in the event of destruction of bituminous binder in asphalt concrete, its stone material will not be displaced like a wave, due to the fact that there will be cement stone binders in it, and the coast protective construction will stay until repair operations.

The use of no-fines concrete here allows to automate the process and reduce the volume of the loaded material due to prior manufacturing of plates.

Taking into account the possibility of using standard cranage, ensuring the plates sustainability for the impact of the ice field and wave loads, it is proposed to use the plates of $1.5 \times 1.5 \times 0.5$ planned dimensions, with a weight of one plate varying within $2.02 \pm$ 0.05 tones. Planned dimensions of asphalt plates allow to lay them out as model units.

\section{Conclusions}

The research yielded the following conclusions:

- to protect the coastal slope, we offer a new technology that involves the use of asphalt concrete together with no-fines concrete;

- the technology includes the following operations: first making a skeleton of no-fines concrete and then washing-down with bituminous materials by a hot procedure;

- advantages of the proposed technology are found, i.e. washing-down with bituminous materials makes it possible to get the final product in a short time, because the asphalt quickly cools and becomes solid. To warm bituminous material up and to deliver it to the point of laying, standard equipment (like bitumen heaters used in road construction) is used. Additional processing of no-fines concrete with bitumen material will significantly increase frost resistance of the final product. It will allow the use of stone materials with frost resistance of 50 cycles and less. Standardizing temperature and place of bituminous material delivery, it is possible to make a layer of drainage and drainage water release that will reduce the thickness of the bind and also reduce costs.

- conducted laboratory studies made it possible to develop a method of appointing technological temperature for the process of bitumen laying;

- in accordance with the methodology for no-fines concrete with $80-120 \mathrm{~mm}$ aggregate we found the appropriate technological temperature of use, that is $170-180{ }^{\circ} \mathrm{C}$.

\section{References}

1. F.F. Tsiter, Using of asphalt coatings to strengthen banks (Strojzdat, Moscow, 1964).

2. I.U. Vizzer, E. Shoenian, F.F. Poskit, «Technical progress in design and construction of high dams», Proceedings of Xth International Congress on High Dams (Energy, Moscow, 1975).

3. Ir. P. A. van de Velde, The use of asphalt in hydraulic engineering (1985).

4. H. Saxegaard, Hydraulic Engineering 12 (2003).

5. P. Londe, M. Lino, Water Power and Dam Construction 2 (1992).

6. A.A. Mikhasek, A.A. Smyvalov, Scientific Survey 14, 102-109 (2015).

7. M.I. Balzannikov, A.A. Mikhasek, Procedia Engineering 91, 183-187 (2014).

8. A.A. Mikhasek, M.V. Rodionov, Procedia Engineering 91, 82-88 (2015).

9. A.A. Mikhasek, Scientific Survey 14, 67-72 (2016).

10. A.A. Mikhasek, M.V. Rodionov, V.A. Seliverstov, Y.E. Senitsky, MATEC Web of Conferences 86 (2016).

11. M.I. Balzannikov, M.V. Rodionov, V.A. Seliverstov, Urban Construction and Architecture 1, 100-105 (2011) 